\title{
Efeitos da iniciação esportiva no desenvolvimento motor e psíquico de crianças em várias faixas etárias: Uma revisão sistemática
}

\author{
Effects of sports initiation on the motor and psychic development of children in various age groups:
} A review systematic

Efectos de la iniciación deportiva sobre el desarrollo motor y psíquico de niños de varios grupos de edad: Una revisión sistemática

Recebido: 28/06/2021 | Revisado: 06/07/2021 | Aceito: 08/07/2021 | Publicado: 18/07/2021

\author{
Rariane Kelly de Moura Cunha \\ ORCID: https://orcid.org/0000-0003-0138-0845 \\ Centro Universitário Santo Agostinho, Brasil \\ E-mail: rariane.kelly1@gmail.com \\ Lucas Gabriel De Pereira Carvalho \\ ORCID: https://orcid.org/0000-0001-9556-1051 \\ Centro Universitário Santo Agostinho, Brasil \\ E-mail: lucaschiupetaa@icloud.com \\ Mauro Fernando Lima da Silva \\ ORCID: https://orcid.org/0000-0001-8866-1855 \\ Centro Universitário Santo Agostinho, Brasil \\ E-mail: maurolima@unifsa.com
}

\begin{abstract}
Resumo
Introdução: A iniciação esportiva além de promover hábitos saudáveis, é importante para a formação e desenvolvimento das capacidades de desempenho psíquico e motor da criança. Objetivo : verificar qual o nível de desenvolvimento motor e psíquico de crianças de diferentes faixas etárias participantes de programas esportivos de iniciação. Métodos: Este estudo trata- se de uma revisão sistemática, as buscas foram realizadas nas bases: Science Direct, Pubmed, BVS, utilizando os descritores: desenvolvimento motor; desenvolvimento psíquico; crianças; iniciação esportiva. A busca na base de dados ocorreu entre janeiro e maio de 2021. Resultados: Foram encontrados no total 196 artigos sobre o tema abordado, nas línguas português, espanhol e inglês. Após a aplicação dos critérios estabelecidos para a pesquisa e leitura dos artigos, apenas 05 foram selecionados por estarem de acordo com o objetivo da pesquisa. Foram obtidas diferenças significativas nos estudos que realizou a correlação do desenvolvimento motor a participação em programas de iniciação esportiva. Conclusão: A iniciação esportiva parece exercer um papel importante no desenvolvimento motor e psicofísico de crianças em várias faixas etárias.
\end{abstract}

Palavras-chave: Desenvolvimento; Psíquico motor; Criança; Iniciação esportiva.

\begin{abstract}
Introduction: In addition to promoting healthy habits, sports initiation is important for the formation and development of the child's psychic and motor performance abilities. Objective: to verify the level of motor and mental development of children of different age groups participating in sports initiation programs. Methods: This study is a systematic review, searches were performed in the following databases: Science Direct, Pubmed, BVS, using the descriptors: motor development; psychic development; kids; sports initiation. The search in thedatabase took place between January and May 2021. Results: A total of 196 articles were found on the topic discussed, in Portuguese, Spanish and English. After applying the criteria established for the research and reading of the articles, only 05 were selected for being in accordance with the research objective. Significant differences were obtained in studies that performed the correlation between motor development and participation in sports initiation programs. Conclusion: Sport initiation seems to play an important role in the motor and psychophysical development of children in various age groups.
\end{abstract}

Keywords: Development; Psychic motor; Child; Sports initiation.

\section{Resumen}

Introducción: Además de promover hábitos saludables, la iniciación deportiva es importante para la formación y desarrollo de las capacidades de desempeño psíquico y motor del niño. Objetivo: verificar el nivel de desarrollo motor y mental de los niños de diferentes grupos de edad que participan en programas de iniciación deportiva. Métodos: Este estudio es una revisión sistemática, se realizaron búsquedas en las siguientes bases de datos: Science Direct, Pubmed, BVS, utilizando los descriptores: desarrollo motor; desarrollo psíquico; niños; iniciación deportiva. La búsqueda en la 
base de datos se realizó entre enero y mayo de 2021. Resultados: Se encontraron un total de 196 artículos sobre el tema tratado, en portugués, español e inglés. Luego de aplicar los criterios establecidos para la investigación y lectura de los artículos, solo se seleccionaron 05 por estar de acuerdo con el objetivo de la investigación. Se obtuvieron diferencias significativas en estudios que realizaron la correlación entre desarrollo motor y participación en programas de iniciación deportiva. Conclusión: La iniciación al deporte parece jugar un papel importante en el desarrollo motor y psicofísico de los niños en varios grupos de edad.

Palabras clave: Desarrollo; Motricidad psíquica; Niños; Iniciación deportiva.

\section{Introdução}

A iniciação esportiva além de promover hábitos saudáveis, é importante para a formação e desenvolvimento das capacidades de desempenho psíquico e motor da criança (Wick; Kriemler; Granacher, 2021). Atrasos nas etapas de maturação biológica e desenvolvimento psicofisiológico de crianças, contribuem efetivamente para possível evasão escolar e frustrações causadas por práticas esportivas não direcionadas (Ricci et al, 2021).

Tem sido sustentado que vinculação, relativamente estáveis ao longo do desenvolvimento psíquico e motor, parecem influenciar o modo como os indivíduos respondem a situações de stress na vida adulta (Sarti et al., 2021). O incentivo dos pais e de profissionais de educação física, pode ser de grande importância, pois acrescenta suporte emocional para a criança, que provavelmente irá desenvolver processos cognitivos e motores de forma satisfatória. (Haitao; Sbeih; Shibly, 2021).

A contribuição de modalidades esportivas para a criança é de fundamental importância, pois as mesmas desenvolvem todas as capacidades necessárias para sua evolução motora (Suggate; Martzog, 2021). Essas capacidades são: correr, pular, saltar, galopar, saltitar, alcançar, agarrar, soltar que são simples padrões de movimento importantes para a sobrevivência do indivíduo (Rodriguez-Negro et al., 2021).

A prática esportiva vem demonstrando ao longo dos anos seus efeitos benéficos inerentes as capacidades motoras e psíquica também (Sparks et al., 2021). Recentemente, foi apresentado ações e contribuições da prática esportiva na infância intimamente relacionada ao desenvolvimento das capacidades cognitivas, tanto em alterações estruturais, quanto funcionais do sistema nervoso (Giacomo et al., 2021)

Ao salientar o efeito funcional do esporte nos domínios cognitivos, estes estão relacionados aos componentes das funções executivas que são responsáveis por planejar, criar, organizar e monitorar nossas ações motoras e psíquicas (Pereira et al. 2021). Tais funcionalidades aparecem em três componentes interligados: controle inibitório, memória de trabalho e flexibilidade cognitiva (Lachambre et al. 2021). Deste modo o objetivo da presente pesquisa foi verificar qual o nível de desenvolvimento motor e psíquico de crianças de diferentes faixas etárias participantes de programas esportivos de iniciação.

\section{Metodologia}

\subsection{Tipo de pesquisa}

Este estudo trata-se de uma revisão sistemática, com base em artigos eletrônicos indexados extraídos nas bases Plataforma da Elsevier - Science Direct, Biblioteca Nacional de Medicina dos Estados Unidos - PUBMED, Biblioteca Virtual em Saúde (BVS), utilizando os descritores: desenvolvimento; psicofiológico; motor; crianças; iniciação esportiva.

Foram considerados os seguintes critérios de inclusão:

i. estudos experimentais, pré-experimentais, quase-experimentais, que realizaram processo de intervenção por meio da correlação das práticas em programas de iniciação esportiva e o desenvolvimento motor;

ii. estudos que verificaram através de ensaios clínicos o desenvolvimento psicofisiológico e motor em crianças a partir da prática de atividade física em programas de iniciação esportiva;

iii. população-alvo composta por crianças praticantes de atividade física em programas de iniciação esportiva;

iv. período de publicação de 2016 a 2021; 
v. publicações em inglês, português e espanhol.

E os critérios de exclusão foram: artigos que não se encaixavam em nenhum dos critérios acima citados:

A seleção dos estudos foi realizada, então, em três etapas:

$1^{\text {a }}$ etapa - leitura dos títulos;

$2^{\mathrm{a}}$ etapa - leitura dos resumos dos artigos selecionados na $1^{\mathrm{a}}$ etapa;

$3^{\mathrm{a}}$ etapa - leitura na íntegra dos artigos selecionados na $2^{\mathrm{a}}$ etapa, e inclusão de outros estudos, contidos nas referências destes artigos, capazes de corresponder aos critérios de inclusão, independente do periódico de publicação.

A busca na base de dados ocorreu entre janeiro e maio de 2021.

\subsection{Estratégia de busca e elegibilidade}

A estratégia PICO foi usada para ajudar a construir a questão norteadora da pesquisa e a seleção do estudo. A estratégia PICO representa a sigla para População, Intervenção, Comparação e Out come (desfecho) (Santos; Pimenta; Nobre, 2007)Quadro 1. Estudos publicados entre 2016 e 2021 foram considerados na revisão.

Quadro 1: Estratégia PICO.

\begin{tabular}{|l|l|l|c|}
\hline $\begin{array}{l}\text { 1. POPULAÇÃO } \\
\text { (PATIENT/PROBLEM) }\end{array}$ & $\begin{array}{l}\text { 2. INTERVENÇÃO } \\
\text { (INTERVENTION) }\end{array}$ & $\begin{array}{l}\text { 3. CONTROLE } \\
\text { (COMPARISION) }\end{array}$ & $\begin{array}{c}\text { 4. DESFECHO } \\
\text { (OUTCOME) }\end{array}$ \\
\hline $\begin{array}{c}\text { Crianças de 6 a } \\
7 \text { anos }\end{array}$ & Iniciação esportiva & $\begin{array}{c}\text { Crianças sem } \\
\text { acesso a } \\
\text { iniciação } \\
\text { esportiva }\end{array}$ & $\begin{array}{c}\text { Melhora do } \\
\text { desenvolvimento motor }\end{array}$ \\
\hline
\end{tabular}

PERGUNTA: A iniciação esportiva melhora o desenvolvimento psíquico e motor na infância?

Fonte: Autores (2021), adaptado de Santos; Pimenta; Nobre (2007)

Quadro 2: Definição de termos e entretermos.

\begin{tabular}{|c|c|}
\hline BASE PESQUISADA & TERMOS E ENTRETERMOS \\
\cline { 1 - 1 } Science Direct & $\begin{array}{c}\text { Iniciação esportiva AND criança AND desenvolvimentomotor } \\
\text { AND psíquico OR child AND motor developmentAND sports } \\
\text { initiation AND psychic }\end{array}$ \\
\cline { 1 - 1 } PUBMED & \\
\hline
\end{tabular}

Fonte: Autores (2021), adaptado de Santos; Pimenta; Nobre (2007)

\section{Resultados e Discussão}

Após o termino das buscas nas bases de dados Plataforma da Elsevier - Science Direct, Biblioteca Nacional de Medicina dos Estados Unidos - PUBMED, Biblioteca Virtual em Saúde (BVS) foram encontrados um total de 196 artigos sobre otema abordado. Após a aplicação dos filtros e após o rastreamento e leitura dos títulos56 foram obtidos, leitura de título e resumo 28, 13 foram excluídos após a leitura de texto completo por estarem fora do escopo da pesquisa, restando somente 15 após análise de síntese quantitativa (03) e qualitativa (2) todos esses artigos, sendo apenas05 selecionados por estarem de acordo com os critérios de inclusão e exclusão e podem ser observados no fluxograma (Quadro 1) .

Em todos os trabalhos analisados os resultados indicam que os programas de intervenção associados a atividades 
esportivas voltadas para o público infantil de várias faixas etárias, induziram melhorias nos níveis de conhecimento declarativo (nível de desenvolvimento motor e psíquico proporcional e esperado para a idade) e procedimental (competência no planejamento e na realização de tarefas). (Gamero,et al., 2021; Isik; Kiliç, 2021; Nardello; Bertucco; Cesari, 2021; Neville, et al., 2021; Rață; Rață; Rață, 2021).

\section{Elegibilidade dos estudos}

Figura 1 - Fluxograma da seleção dos artigos (Prisma Flow).
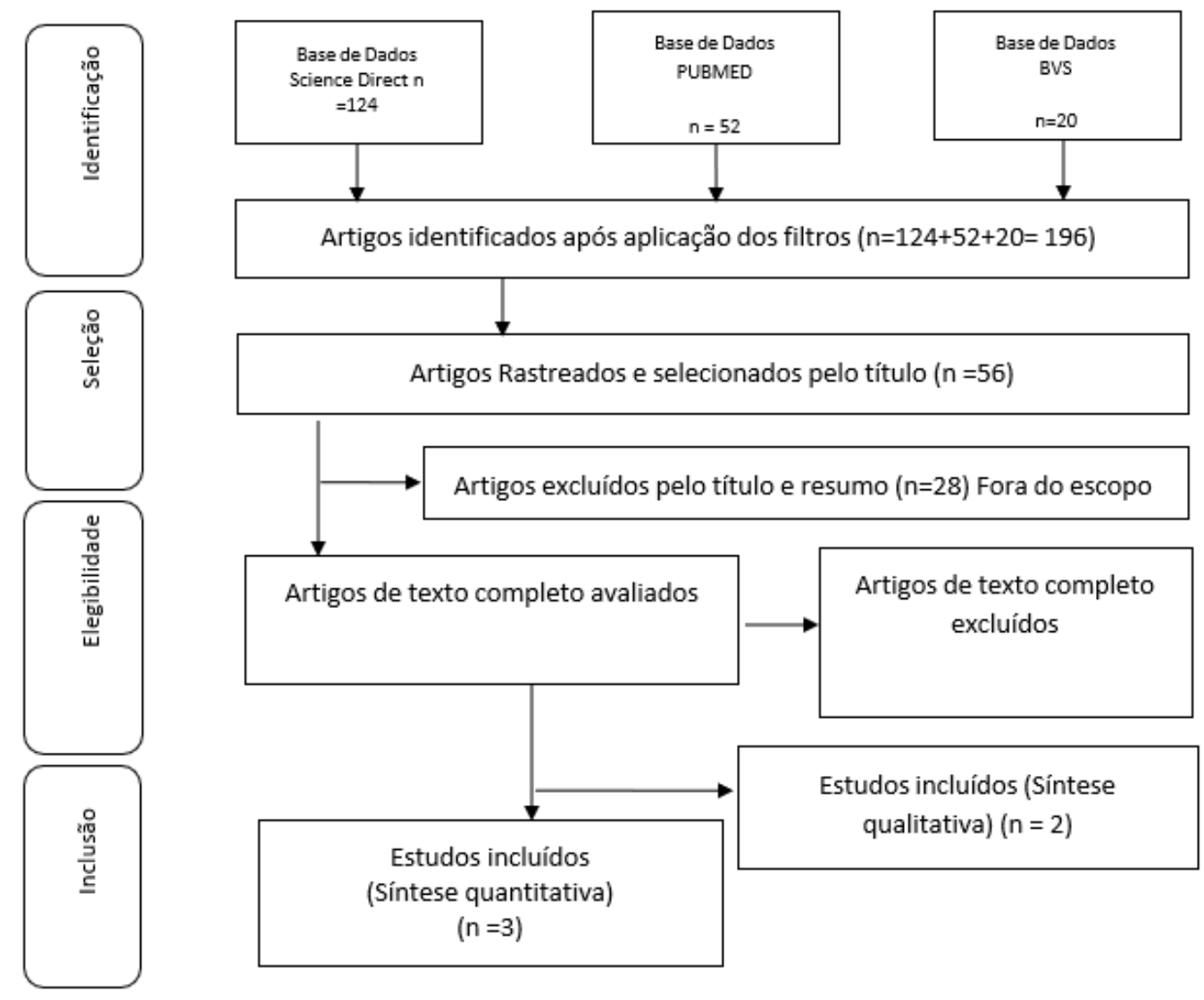

Fonte: Autores (2021), adaptado de Moher et al., 2009.

Trabalhos com direcionamento mais específico, como foi revelado na aplicação das técnicas de tiro com hemsball (uma variação técnica do tiro livre do basquete) tiveram um efeito significativo nos níveis de proficiência motora fina de crianças com deficiência auditiva, incluindo precisão motora fina, integração motora fina e destreza manual (Isik; Kiliç, 2021).

Resultados similares foram encontrados em programas de iniciação de futebol e natação, desta vez com crianças entre 11 e 12 anos, onde e quando comparados mostrou que as crianças submetidas ao programa voltado para o futebol superaram os nadadores por apresentar maior estabilidade e menor número de quedas durante a competição, onde o controle proativo era principalmente necessário para a manutenção da postura (Nardello; Bertucco; Cesari, 2021).

Outros aspectos relevantes reportados nesta revisão, são de que, com a idade de 1 ano foi possível observar atrasos no desenvolvimento motor e psíquico (dificuldade comportamentais e pró-sociais, ou seja, favoráveis ao convívio com os da mesma idade) esperado para a faixa etária e que pode permanecer até os 03 anos caso não tenha envolvimento com a prática esportiva, situação contraria a mostrada quando foi comprado este grupo com o grupo ativo em programas de esporte de iniciação (Neville, et al., 2021). 
Em uma pesquisa experimental com Atletismo indoor, com crianças de 7 a 8 anos, mostrou que os objetivos citados acima foram atingidos utilizando exercícios de jogo, que visavam aprender os elementos básicos da escola técnica de corrida, salto e lançamento, jogos de movimento, revezamentos, pistas de esportes, ao final dos trabalhos foram reportados a melhoria ainda da coordenação geral e na velocidade de deslocamento e de reação da crianças analisadas (Raţă; Rață; Rață, 2021).

Quadro 1 - Síntese dos Artigos incluídos na Revisão Sistemática. PI, 2021.

\begin{tabular}{|c|c|c|c|}
\hline $\begin{array}{l}\text { AUTOR, } \\
\text { DATA }\end{array}$ & OBJETIVO & METODO & RESULTADOS \\
\hline $\begin{array}{l}\text { Gamero, et } \\
\text { al., } 2021 .\end{array}$ & \begin{tabular}{llr}
$\begin{array}{l}\text { Comparar a } \\
\text { conhecimentos }\end{array}$ & \multicolumn{2}{c}{ aquisiçãa declarativos } \\
procedimentais & e após \\
implantação & de diversos \\
programas de intervenção no \\
basquetebol escolar, de acordo \\
com a metodologia e experiência \\
prévia dosalunos.
\end{tabular} & $\begin{array}{l}-55 \text { alunos do sexto ano do ensino } \\
\text { fundamental, distribuídos em três turmas. } \\
\text {-Cada grupo participou de um programa de } \\
\text { intervenção diferente: abordagem de jogos } \\
\text { táticos (TGA), instrução direta (DI) ou } \\
\text { unidade de basquete do professor de serviço } \\
\text { (STBU). } \\
\text {-O nível de conhecimento foi medido por } \\
\text { meio do Teste de Conhecimento } \\
\begin{array}{l}\text { Declarativo e Processual no Basquete } \\
\text { (TDPKB). }\end{array} \\
\text {-Uma análise descritiva foi realizada para } \\
\text { determinar as características dos } \\
\text { participantes. } \\
\text {-Uma ANOVA fatorial foi } \\
\text { subsequentemente aplicada em duas fases } \\
\text { (pré-teste e pós-teste) para amostras } \\
\text { independentes para comparar o nível de } \\
\text { conhecimento entre os diferentes grupos, e } \\
\text { um teste t para amostras relacionadas foi } \\
\text { realizado para comparar o nível de } \\
\text { conhecimento pré e pós dentro de cada } \\
\text { grupo. } \\
\text {-Em seguida, uma ANOVA fatorial e um } \\
\text { teste de medidas repetidas foram realizados } \\
\text { para determinar o efeito da metodologia e } \\
\text { da experiência sobre o conhecimento dos } \\
\text { alunos. }\end{array}$ & $\begin{array}{l}\text {-Os resultados indicam que os programas } \\
\text { de intervenção TGA, DI e STBU } \\
\text { induziram melhorias nos níveis de } \\
\text { conhecimento declarativo } \\
\text { procedimental em todas as turmas, com } \\
\text { os alunos que participaram do programa } \\
\text { TGA alcançando níveis mais elevados de } \\
\text { conhecimento declarativo. } \\
\text {-Por fim, o efeito da ausência de } \\
\text { experiência prática foi identificado como } \\
\text { fator determinante para melhorias. } \\
\text {-Os alunos que não haviam } \\
\text { praticadobasquete anteriormente } \\
\text { alcançaram níveis mais elevados de } \\
\text { conhecimento com o programa de } \\
\text { intervenção TGA. }\end{array}$ \\
\hline $\begin{array}{l}\text { Isik; Kiliç, } \\
2021 .\end{array}$ & $\begin{array}{l}\text { Determinar o efeito de técnicas de } \\
\text { tiro hemsball (variação do tiro } \\
\text { livre do basquetebol) na } \\
\text { proficiênciamotora fina e destreza } \\
\text { manual em crianças com } \\
\text { deficiência auditiva na faixa } \\
\text { etária de } 7 \text { anos. }-11 .\end{array}$ & $\begin{array}{l}\text {-Estudo, que foi desenhado em um modelo } \\
\text { de pré-teste - pós-teste e controle } \\
\text { experimental; } \\
\text { - O grupo de estudo foi composto por } 26 \\
\text { crianças ( } 13 \text { para o grupo experimental + } 13 \\
\text { para o grupo controle) com idades entre } 7 \text { - } \\
11 \text { anos com perda auditiva acima de } 56 \mathrm{~dB} \\
\text { e sem segunda deficiência e histórico } \\
\text { esportivo. } \\
\text {-As crianças do grupo experimentalforam } \\
\text { submetidas a um programa de } \\
\text { treinamentopara }\end{array}$ & $\begin{array}{l}\text {-Resultado do estudo, foi revelado que as } \\
\text { técnicas de tiro com hemsball tiveram um } \\
\text { efeito significativo nos níveis de } \\
\text { proficiência motora fina de crianças com } \\
\text { deficiência auditiva, incluindo precisão } \\
\text { motora fina, integração motora fina e } \\
\text { destrezamanual. } \\
\text {-Além disso, foi determinado que o efeito } \\
\text { das técnicas de tiro de hemsball na } \\
\text { proficiência motora não diferiu } \\
\text { significativamente de acordo com o } \\
\text { gênero e que a aplicação realizada } \\
\text { teve }\end{array}$ \\
\hline & & $\begin{array}{l}\text { Aperfeiçoamento da motricidade fina e } \\
\text { destreza manual três dias por semana, uma } \\
\text { hora por dia durante } 10 \text { semanas, } \\
\text { envolvendotécnicas de tiro com hemsball. }\end{array}$ & efeitos positivos em meninase meninos. \\
\hline
\end{tabular}




\begin{tabular}{|c|c|c|c|}
\hline & & $\begin{array}{l}\text {-A proficiência motora fina e destreza } \\
\text { manual de crianças com deficiência auditiva } \\
\text { forammedidas pelos três subtestes (precisão } \\
\text { motora fina, integração motora fina, } \\
\text { destreza manual) do Teste de Proficiência } \\
\text { Motora de Bruiniks-Oseretsky (BOT-2). } \\
\text {-Na pesquisa, o grupo experimento-controle } \\
\text { e as medidas (pré-teste - pós- teste) foram } \\
\text { comparados com o teste t de amostras } \\
\text { independentes e o teste t de amostras } \\
\text { pareadas. }\end{array}$ & \\
\hline $\begin{array}{l}\text { Nardello; } \\
\text { Bertucco; } \\
\text { Cesari, } \\
2021\end{array}$ & $\begin{array}{l}\text { Investigou se existe diferença no } \\
\text { controle reativo e proativo para } \\
\text { esportes considerados habilidades } \\
\text { abertas oufechadas dominadas. }\end{array}$ & $\begin{array}{l}\text {-Dezesseis atletas jovens (11-12 anos) (oito } \\
\text { jogadores defutebol e oito nadadores) } \\
\text {-Foram convidados a participar de duas } \\
\text { competições de jogos que exigiam um tipo } \\
\text { de controlereativo e outro proativo. } \\
\text {-Por meio de análise cinemática (ou seja, } \\
\text { tempo e duração do movimento) e dinâmica } \\
\text { através da plataforma de força (ou seja, } \\
\text { Ajustes Posturais } \\
\text { Antecipatórios, APAs),avaliamos o nível de } \\
\text { habilidade e estabilidade em reagir e } \\
\text { antecipar ações. }\end{array}$ & $\begin{array}{l}\text {-Os resultados indicaram que os jogadores } \\
\text { de futebol superaram os nadadores por } \\
\text { apresentar maior estabilidade e menor } \\
\text { número de quedas durante a competição, } \\
\text { onde o controle proativo era } \\
\text { principalmente necessário. } \\
\text {-Os jogadores de futebol conseguiram } \\
\text { chegar a esse resultado antecipando ações } \\
\text { por meio de APAs bem modulados. } \\
\text {-Ao contrário, durante a competição em } \\
\text { que o controle reativo era principalmente } \\
\text { necessário, os desempenhos eram } \\
\text { comparáveis entre os grupos. Portanto, o } \\
\text { desenvolvimento do controle de ações } \\
\text { específicas já está estabelecido aos } 11-12 \\
\text { anos de idade e é potencializado pela } \\
\text { especificidade do treinamento. }\end{array}$ \\
\hline $\begin{array}{l}\text { Neville, et } \\
\text { al., } 2021 .\end{array}$ & $\begin{array}{l}\text { Explorar se as associações entre } \\
\text { atrasos no desenvolvimento no } \\
\text { primeiro ano de vida e resultados } \\
\text { psicossociais em crianças pré- } \\
\text { escolares são afetadas pela } \\
\text { participação em esportes } \\
\text { organizados. }\end{array}$ & $\begin{array}{l}\text { Os dados foram obtidos da coorte infantil } \\
\text { do projeto Growing Up in Ireland. Os pais } \\
\text { relataram sobre o desenvolvimento infantil } \\
\text { (Ages and Stages Questionnaire) aos } 1 \text { ano } \\
\text { de idade, características psicossociais } \\
\text { (Strengths and Difficulties Questionnaire) } \\
\text { aos } 3 \text { e } 5 \text { anos,e envolvimento em esportes } \\
\text { organizados aos } 5 \text { anos. Os }\end{array}$ & $\begin{array}{l}\text { - Com a idade de } 1 \text { ano, } 15 \% \text { da coorte foi } \\
\text { classificada como tendo atrasosno } \\
\text { desenvolvimento. } \\
\text {-Essas crianças exibiram maisdificuldades } \\
\text { comportamentais }(0,55, \pm 0,27 \text {; diferença } \\
\text { média, limites de confiança de } \pm 95 \% \\
[C L]) \quad(P<0,0001) \text { e menos } \\
\text { comportamentos pró-sociais }(-0,54, \pm \\
0,11)(P<0,0001) \text { aos } 3 \text { anos de idade } \\
\text { anos. }\end{array}$ \\
\hline & & $\begin{array}{l}\text { Dados foram analisados usando modelos } \\
\text { mistos. }\end{array}$ & 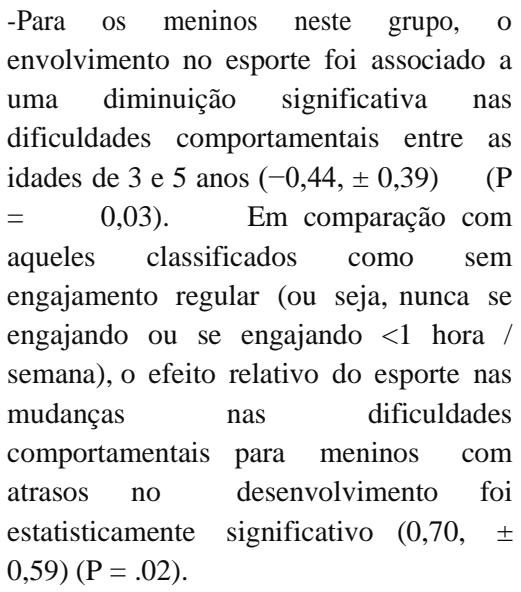 \\
\hline & & & $\begin{array}{l}\text {-A participação em esportes não foi } \\
\text { associada a mudanças significativas nas } \\
\text { dificuldades comportamentais para } \\
\text { meninas, ou uma mudança significativa } \\
\text { nos comportamentos pró-sociais para }\end{array}$ \\
\hline
\end{tabular}




\begin{tabular}{|c|c|c|c|}
\hline & & & meninos ou meninas. \\
\hline $\begin{array}{l}\text { Rață; } \\
\text { ață; } \\
\text { ață,2021. }\end{array}$ & $\begin{array}{l}\text { Evidenciar o aprimoramento das } \\
\text { habilidades de coordenação e } \\
\text { velocidade das crianças por meio } \\
\text { de um programa de treinamento } \\
\text { operacional durante as aulas de } \\
\text { treinamento esportivo, baseado na } \\
\text { aquisição de habilidades motoras } \\
\text { específicas para eventos } \\
\text { esportivos. }\end{array}$ & $\begin{array}{l}\text {-Esse programa operacional foi aplicado } \\
\text { durante } 4 \text { meses, três vezes por semana, em } \\
\text { uma amostra de } 20 \text { crianças de } 7 \text { a } 8 \text { anos. } \\
\text {-A evolução das habilidades de coordenação } \\
\text { e velocidade foi avaliada pelo Teste Touch- } \\
\text { the-Plates, pelo teste de Denisiuk e pelo } \\
\text { teste de sprintde } 20 \mathrm{~m} \text {, realizados no início e } \\
\text { no final do experimento. }\end{array}$ & $\begin{array}{l}\text {-A pesquisa experimental aconteceu no } \\
\text { Salão de Atletismo do Bacau, uma salade } \\
\text { pista circular de } 200 \mathrm{~m} \text {. } \\
\text {-Para atingir os objetivos foram utilizados } \\
\text { exercícios de jogo, que visavam aprender } \\
\text { os elementos básicos da escola técnica de } \\
\text { corrida, salto e lançamento, jogos de } \\
\text { movimento, revezamentos, pistas de } \\
\text { esportes. } \\
\text {-As conclusões destacam a melhoria da } \\
\text { coordenação e velocidade. }\end{array}$ \\
\hline
\end{tabular}

A participação em esportes não foi associada a mudanças significativas nas dificuldades comportamentais para as crianças estudadas, ou uma mudança significativa nos comportamentos pró-sociais para meninos ou meninas. A participação regular de meninos em esportes pode atenuar algumas das dificuldades comportamentais associadas ao desenvolvimento em idades iniciais, fato este que tende a repercutir na vida futura. A falta de oportunidades para praticar esportes pode afetar negativamente a regulação comportamental de crianças em várias idades da pré-escola as primeiras series do ensino fundamental, principalmente ao que se refere ao desenvolvimento motor (gestos básicos que irão influenciar o nível de atividade física futura) quanto o psíquico (função executiva, essencial no planejamento de tarefas de estudo e/ou trabalho). (Gamero, et al., 2021; Isik; Kiliç, 2021; Nardello; Bertucco; Cesari, 2021; Neville, et al., 2021; Rață; Rață; Rață, 2021).

\section{Conclusão}

Conclui-se que os estudos revisados apresentaram resultados relevantes sobre os efeitos benéficos das práticas introdutórias de modalidades esportivas diversas com planejamento e execução adequado para crianças e pré-púberes, sendo que os componentes mais evidenciados foram às funções executivas e atenção seletiva durante a atividade física sendo estes associados ao melhor desenvolvimento neuropsíquico-motor das crianças. A larga carência de estudos clínicos randomizados, remete a expor a necessidade de novas pesquisas com este formato para melhor elucidar o tema aqui debatido.

\section{Referências}

Garcia, L., \& Ramada, B. (1993). Maturacion sexual e biológica. An Esp Ped, Barcelona, 3, 8.

Gamero, M. G., García-Ceberino, J. M., Ibáñez, S. J., \& Feu, S. (2021). Analysis of declarative and procedural knowledge according to teaching method and experience in school basketball. Sustainability, 13(11), 6012.

Giacomo, R., Maddalena, FD, Arturo, N., Gatti, R., \& Pietro, A. (2021). O papel do mecanismo de espelho na recuperação, manutenção e aquisição de habilidades motoras. Neuroscience \& Biobehavioral Reviews

Giacomo, R., Maddalena, F. D., Arturo, N., Gatti, R., \& Pietro, A. (2021). The role of mirror mechanism in the recovery, maintenance, and acquisition of motor abilities. Neuroscience \& Biobehavioral Reviews.

Haitao, H. A. O., Sbeih, A., \& Shibly, F. H. A. (2021). Physical education and its role in improving the health of college students by active participation and optimization by DEEP learning. Aggression and Violent Behavior, 101628.

Huang, Y. C., \& Malina, R. M. (2002). Physical activity and health-related physical fitness in Taiwanese adolescents. Journal of physiological anthropology and applied human science, 21(1), 11-19. 
Isik, M., \& Kiliç, I. (2021). Effect of Hemsball Shooting on Fine Motor Proficiency in Hearing Impaired Children. International Education Studies, 14(4), 1120 .

Lachambre, C., Proteau-Lemieux, M., Lepage, J. F., Bussières, E. L., \& Lippé, S. (2021). Attentional and executive functions in children and adolescents with developmental coordination disorder and the influence of comorbid disorders: A systematic review of the literature. PloS one, 16(6), e0252043.

Moher, D., Liberati, A., Tetzlaff, J., Altman, D. G., \& Prisma Group. (2009). Preferred reporting items for systematic reviews and meta-analyses: the PRISMA statement. PLoS medicine, 6(7), e1000097.

Nardello, F., Bertucco, M., \& Cesari, P. (2021). Anticipatory and pre-planned actions: A comparison between young soccer players and swimmers. PloS one, 16(4), $\mathrm{e} 0249635$.

Neville, R. D., Guo, Y., Boreham, C. A., \& Lakes, K. D. (2021). Longitudinal association between participation in organized sport and psychosocial development in early childhood. The Journal of Pediatrics, 230, 152-160.

Pereira, S., Garbeloto, F., Guimarães, E., Santos, C., Baxter-Jones, A., Tani, G., ... \& Maia, J. (2021). Physical fitness spurts in childhood: A study in boys. Scandinavian Journal of Medicine \& Science in Sports, 31, 47-55.

Rață, B. C., Rață, M., \& Rață, G. (2021). The Influence of Exercises in Athletics on Teaching Speed and Coordination in 7-8-Year-Old Children. Gymnasium, $21(2), 5-24$

Ricci, A., He, F., Fang, J., Calhoun, S. L., Vgontzas, A. N., Liao, D., \& Fernandez-Mendoza, J. (2021). Maturational trajectories of non-rapid eye movement slow wave activity and odds ratio product in a population-based sample of youth. Sleep Medicine, 83, 271-279.

Rodriguez-Negro, J., Llodio, I., Castillo, D., Romaratezabala, E., \& Yanci, J. (2021). Changes in selected locomotor skills of young runners after one athletic season: Influence of sex and age. International Journal of Sports Science \& Coaching, 17479541211006676.

Santos, C. M. D. C., Pimenta, C. A. D. M., \& Nobre, M. R. C. (2007). A estratégia PICO para a construção da pergunta de pesquisa e busca de evidências. Revista Latino-Americana de Enfermagem, 15(3), 508-511.

Sarti, A. C. (2021). Desenvolvimento de atletas: validação inicial do" Psychological Characteristics of Developing Excellence Questionnaire (version 2)" para a língua portuguesa.

Sparks, K. V., Kavussanu, M., Masters, R. S., \& Ring, C. (2021). Mindfulness, reinvestment, and rowing under pressure: Evidence for moderated moderation of the anxiety-performance relationship. Psychology of Sport and Exercise, 101998.

Suggate, S. P., \& Martzog, P. (2021). Children's sensorimotor development in relation to screen-media usage: A two-year longitudinal study. Journal of Applied Developmental Psychology, 74, 101279.

Wick, K., Kriemler, S., \& Granacher, U. (2021). Effects of a Strength-Dominated Exercise Program on Physical Fitness and Cognitive Performance in Preschool Children. The Journal of Strength \& Conditioning Research, 35(4), 983-990.

Young, A., Healy, S., Silliman-French, L., \& Brian, A. (2021). A Pilot Study of a Parent-Mediated, Web-Based Motor Skill Intervention for Children With wn Syndrome: Project SKIP. Adapted Physical Activity Quarterly, 1(aop), 1-22. 